\title{
CASE REPORT: LUNG NEOPLASIA IN A PATIENT WITH RHEUMATOID ARTHRITIS USING TOCILIZUMAB
}

Letícia Resende Brandão ${ }^{1}$ Eduardo Rabello Laignier², Elizandra Tomazela Laurenti Polito ${ }^{1, \star}$

1.Hospital Estadual Dório Silva, Serra (ES), Brazil; 2.Clínica Radiológica Santa Elisa, Guarapari (ES), Brazil.

*Corresponding author: eliz.polito@gmail.com

\section{BACKGROUND}

We report a case of a patient with rheumatoid arthritis (RA) using tocilizumab who presented mental confusion and hyponatremia, inappropriate ADH secretion syndrome (SIADH), due to lung cancer.

\section{CASE REPORT}

A 68-year-old female, white, retired, with RA diagnosed in 2013, positive rheumatoid factor, and had been on tocilizumab use since 2017 in monotherapy, with disease remission. Previous use of methotrexate, leflunomide, adalimumab. Comorbidities: osteoporosis, bronchiectasis, right knee prosthesis, hypothyroidism. In October 2019, presented mental confusion, lowering of the level of consciousness, and zoster herpes on the face. She was referred to the emergency room and hyponatremia ( $\mathrm{Na}=122)$ was evidenced. She was hospitalized for correction of the hydro electrolytic disorder and diagnostic research, evidencing chest CT imaging (Figure 1) with lung nodule, findings of an uncertain nature and evolutionary potential and highly suggestive of primary injury. Bronchoscopy was performed with bronchoalveolar washes and research of fungus, micobacterium tuberculosis and other mycobacteria, with cytology, cultures and negative PCR test. Lung lesion biopsy: lung adenocarcinoma. Pet scan January 2021: hypermetabolic pulmonary nodule in the right upper apical lobe; absence of other suspicious areas for active neoplastic involvement. Started treatment with osimertinib and maintaining good clinical evolution. From the rheumatological point of view, the patient is taking methotrexate $15 \mathrm{mg} /$ week, folic acid, prednisone $5 \mathrm{mg} /$ day, calcium and vitamin $\mathrm{D}$, denosumab.

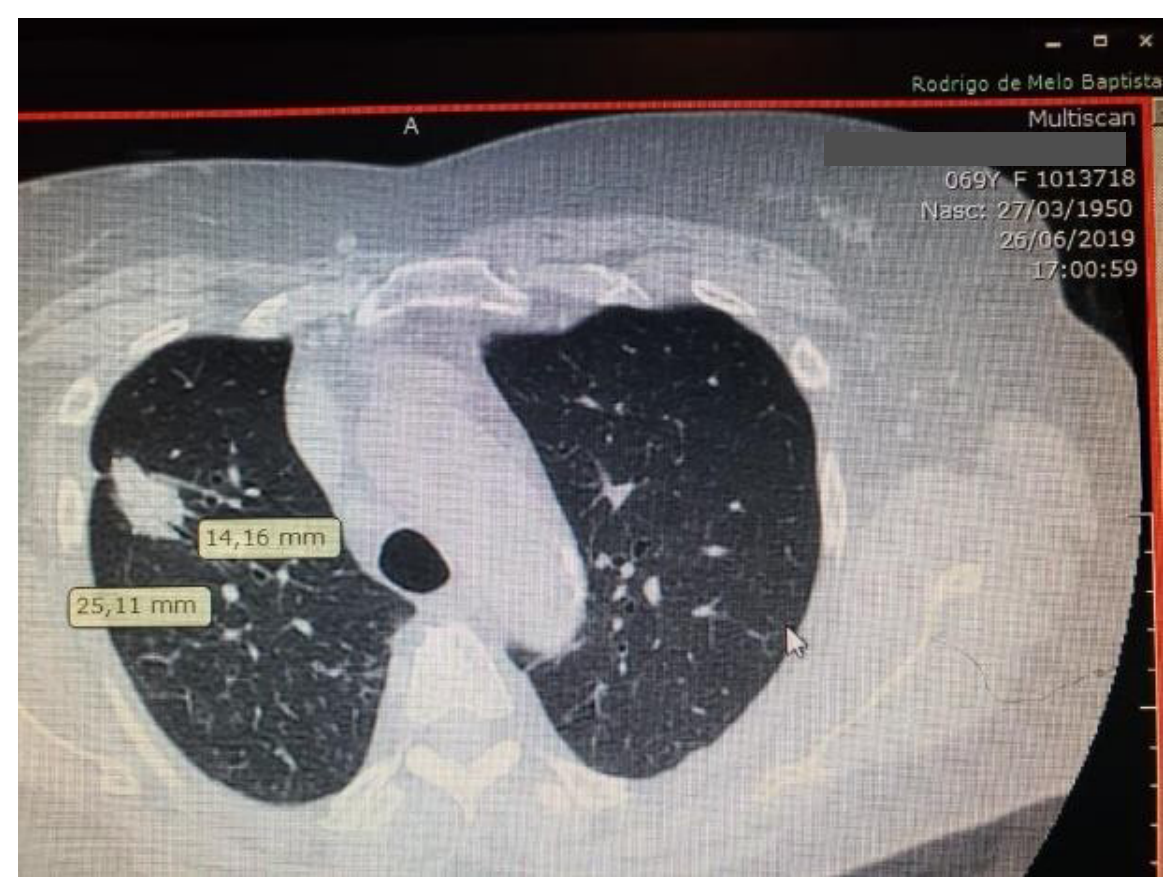

Figure 1. Chest CT June 2019: nodular lesion with soft tissue density, irregular contours and certain pleural retraction in the right upper lobes. 


\section{CONCLUSION}

The incidence of lung cancer in patients with RA is generally increased compared to that of the general population, especially in men over 55 years of age, and in those with felty syndrome. Survival and prognosis for patients with RA who develop lung cancer is worse than for the general population and smoking is a risk factor for mortality. The risk of iatrogenic cancer with the use of immunosuppressive drugs is of great concern, but the assessment of this risk is confused due to the lack of comparable control groups, such as risk assessments of the general population, or even patients with less severe RA. Because the vast majority of RA patients received some type of therapy during the course of the disease, studies on cancer risk and DMARDs generally compare the risk of malignancy in this population of patients to the general population, or the risk of cancer in patients with RA receiving agents not associated with increased risk, such as hydroxychloroquine. Information about cancer risk for patients using tocilizumab is scarce. Until the present time, it has not been associated with an increased risk for malignancy.

\section{KEYWORDS}

Rheumatoid arthritis, Lung cancer, Tocilizumab, SIADH. 